\title{
FNCLCC Sarcoma Grade GX Grade cannot be assessed
}

National Cancer Institute

\section{Source}

National Cancer Institute. FNCLCC Sarcoma Grade GX Grade cannot be assessed. NCI

Thesaurus. Code C160730.

A sarcoma with a GX grade that cannot be assessed. 